Interfaces

\title{
L'esthétique du déchet et du recyclage chez Joseph Cornell
}

\section{Antonia Rigaud}

\section{(2) OpenEdition}

1 Journals

Édition électronique

URL : http://journals.openedition.org/interfaces/884

DOI : 10.4000/interfaces.884

ISSN : 2647-6754

Éditeur :

Université de Bourgogne, Université de Paris, College of the Holy Cross

\section{Édition imprimée}

Date de publication : 15 juillet 2020

Pagination : 75-87

ISSN : 1164-6225

\section{Référence électronique}

Antonia Rigaud, «L'esthétique du déchet et du recyclage chez Joseph Cornell », Interfaces [En ligne],

43 | 2020, mis en ligne le 15 juillet 2020, consulté le 30 décembre 2020. URL : http://

journals.openedition.org/interfaces/884; DOI : https://doi.org/10.4000/interfaces.884

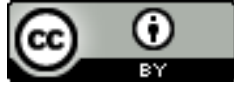

Les contenus de la revue Interfaces sont mis à disposition selon les termes de la Licence Creative Commons Attribution 4.0 International. 


\title{
L'ESTHÉTIQUE DU DÉCHET ET DU RECYCLAGE CHEZ JOSEPH CORNELL
}

\author{
Antonia Rigaud \\ Laboratoire Prismes EA 4398, Université Sorbonne Nouvelle
}

\begin{abstract}
Résumé : Cet article s'intéresse à la manière dont l'artiste Joseph Cornell transforme les détritus de la société de consommation américaine du début du $\mathrm{XX}^{\mathrm{eme}}$ siècle en objets d'art. Derrière une pratique artistique qui évoque d'abord les figure du flâneur et du chiffonnier se dégage une esthétique profondément américaine, qui fait écho à l'esthétique de l'ordinaire définie par Emerson un siècle plus tôt. La thématique du recyclage chez Cornell permet de le situer dans la tradition spécifiquement américaine de la philosophie de l'ordinaire.
\end{abstract}

Mots-clés : Cornell (Joseph), Emerson (Ralph Waldo), Thoreau (Henry David), surréalisme, bricolage, détritus, chiffonnier

Abstract: This article looks at the way Joseph Cornell transforms detritus from early $20^{\text {th }}$ century consumer society into artworks. This gesture evokes the figures of the flâneur and the rag-picker, but ultimately corresponds to the specifically American aesthetics of the low as it was defined by Emerson a century earlier. The theme of recycling allows to situate Cornell within the specifically American tradition of ordinary philosophy.

Keywords: Cornell (Joseph), Emerson (Ralph Waldo), Thoreau (Henry David), Surrealism, bricolage, waste, ragpicker 
Lorsque Ralph Waldo Emerson, le philosophe de la jeune Amérique, appelle à la fin des idoles et à une relation directe à l'univers, il déclare la naissance d'une nouvelle esthétique fondée sur la tabula rasa, sur la destruction des monuments du passé au profit de la célébration d'une esthétique de l'ordinaire, du banal, de ce qu'il appelle « the low $»{ }^{1}$ Emerson appelle ainsi de ses vœux en I837 une nouvelle culture spécifiquement américaine qui serait une culture du banal et de l'anti-monumental. Le philosophe pense une Amérique « innocente », dont la culture ne peut qu'être différente de la culture européenne, et ce discours, lui-même très proche de la philosophie européenne de l'époque, va prendre racine en terre américaine et devenir le socle de la philosophie d'une jeune nation aux ambitions esthétiques et culturelles fondées sur l'ordinaire.

Un peu moins d'un siècle plus tard, Joseph Cornell crée des univers oniriques faits d'objets trouvés, de déchets de la société de consommation américaine recyclés et sublimés en objets d'art qui marqueront l'histoire de l'art des États-Unis. ${ }^{2}$ Cornell est une figure extrêmement ambiguë de l'histoire de l'art et de la culture américaine. Nombres de ses caractéristiques sont celles que l'on attribue aux artistes « outsiders » : Cet artiste autodidacte présente dans son œuvre une vision tout à fait singulière du monde qui exerce pourtant une influence fondamentale sur un grand nombre d'artistes s'inscrivant dans son sillage. Vivant reclus dans la maison familiale de la banlieue de New York, sur Utopia Parkway, un fait largement souligné par la critique, il commence sa carrière loin du monde de l'art mais devient rapidement une figure recherchée des milieux artistiques et le restera tout au long de sa carrière. Sa maison et son atelier furent des lieux de passage pour nombre d'artistes et d'intellectuels qui ont écrit l'histoire de l'avant-garde américaine au XX ${ }^{\text {ème }}$ siècle, tels que Robert Motherwell, Susan Sontag, ou la performeuse Carolee Schneemann. On est donc face à une figure à la fois marginale et prescriptrice, dont l'esthétique a marqué des générations d'artistes, peut-être parce qu'elle fait le lien entre l'esthétique de l'ordinaire célébrée par Emerson et les expérimentations artistiques qui marqueront le $\mathrm{XX}^{\text {ème }}$ siècle américain. L'œuvre de Cornell semble être un point de jonction dans la culture américaine entre un premier moment de célébration de l'ordinaire comme esthétique de la jeune nation au $\mathrm{XIX}^{\mathrm{eme}}$ siècle et les réponses artistiques à la société de consommation au cours du $\mathrm{XX}^{\mathrm{ème}}$ siècle.

Cornell crée des assemblages, des collages et des films, construisant dans ces différents médias des univers clos, faisant de chacune de ses œuvres des microcosmes oniriques dont on oublie parfois,

Ralph Waldo Emerson, "The American Scholar": "I embrace the common, I explore and sit at the feet of the familiar, the low. Give me insight into to-day, and you may have the antique and future worlds". (Emerson 67)

2. Les sites du Museum of Modern Art de New York et du Smithsonian American Art Museum présentent des sélections détaillées des œuvres de Joseph Cornell qui peuvent être consultées en ligne : https://www.moma.org/artists/I247. https://americanart.si.edu/artist/joseph-cornell-995 (page consultée le 20 juin 2020). 
face à l'effet de magie qu'ils produisent, qu'ils sont créés à partir d'images et d'objets épars, glanés au fil des déambulations new-yorkaises de l'artiste. À l'instar du flâneur européen, Cornell arpente les rues de la ville moderne dont il observe minutieusement les images et les détritus qu'il collectionne ; il représente aussi en cela l'image du chiffonnier qui amasse les détritus allant de pair avec les nouveaux modes de production de masse. Marc Berdet analyse la double fascination de Walter Benjamin pour ces deux figures de la modernité en rappelant les deux modalités esthétiques du flâneur et du chiffonnier. Il affirme ainsi : " Le flâneur est désordonné, impulsif et ambigu ; le chiffonnier est méthodique, réfléchi et implacable. Le flâneur s'intéresse aux étoiles, aux symboles de souhait, à l'allégorie et aux 'rêvoirs' ; le chiffonnier aux rêves brisés qui gisent sur le macadam et non pas dans les étoiles, aux objets qu'il peut y 'collectionner' selon un ordre qui subvertit le fétichisme de la valeur d'échange » (Berdet 425). Cornell se situe sur la ligne de crête entre ces deux modalités d'appréhension du monde et semble en proposer une synthèse dans des œuvres qui s'ouvrent aux étoiles à partir des « rêves brisés qui gisent sur le macadam ».

Présent dans les galeries new-yorkaises dès les années 30, il devient, en dépit de ses excentricités et de sa marginalité géographique, une figure incontournable de l'avant-garde américaine. Ses boîtes, ces mondes minuscules, se situent sur une échelle bien éloignée de celle des vastes toiles de l'expressionnisme abstrait, telles celles de De Kooning, Mark Rothko ou plus tard Rauschenberg, mais son art de la récupération et du recyclage a exercé une influence certaine sur l'avant-garde américaine qui s'est exportée tout au long du XX ${ }^{\text {ème }}$ siècle. Cornell définit en effet une esthétique où les images et les objets ordinaires de l'Amérique sont recyclés en œuvres d'art, dans une approche à la croisée de celle du flâneur et du chiffonnier. Il semble en cela faire écho à l'appel d'Emerson pour une culture et une esthétique de l'ordinaire, transformant l'ordinaire en œuvre d'art. Mais l'artiste répond à cette philosophie de l'ordinaire ancrée dans le XIX ${ }^{\mathrm{eme}}$ siècle par la méthode du collage que le critique David Banash définit comme la modalité esthétique même de l'art au XX ${ }^{\text {ème }}$ siècle, ce qui permet de le situer à la croisée du $X X^{\text {ème }}$ et du XX ${ }^{\text {ème }}$ siècles, d'en faire une figure syncrétique de la culture américaine.

Le geste de la tabula rasa est particulièrement paradoxal chez Cornell : son élan créateur ne se situe pas dans la destruction mais plutôt dans l'appropriation d'objets détruits et rejetés, d'objets sans valeur, dont il fait les fondements d'un art qui met en question la notion même de valeur. Il rapproche aussi le geste créateur du bricolage, appelant à une réévaluation des codes de la création artistique.

Cornell commence à accumuler des objets en sillonnant les rues de New York dès les années 20 faisant son matériau de la culture des magazines et des objets sans valeur marchande issus de la production en série ou du divertissement populaire. Ces objets avaient déjà bien sûr fait leur apparition dans l'art avant Cornell, avec les cubistes notamment, mais là où les cubistes faisaient des collages rendant compte du monde contemporain, Cornell, lui, cherchait dans ses collages à s'éloigner du réel, 
à y ajouter une dimension quasiment spirituelle. L'artiste vise bien à transcender ces objets sans valeur pour les assembler en des boîtes qui poétisent le banal de la société américaine du début du XX ${ }^{\mathrm{eme}}$ siècle. Il met en perspective les notions de création et de destruction en suggérant une spécificité de ces notions dans le contexte états-unien.

Cornell pratique une poésie de l'accumulation avec des œuvres qui semblent directement issues des modes de production de masse de la modernité du début du $\mathrm{XX}^{\mathrm{ème}}$ siècle. Mary Ann Caws rappelle qu'il envisage ses boîtes comme l'expression d'une « métaphysique de l'éphémère » ${ }^{3}$ (Caws I36), mettant ainsi en question l'idée de pérennité et de valeur de l'œuvre d'art. En dernier lieu, les boîtes de Cornell nous font voir ou redécouvrir l'ordinaire, dans un geste qui permet de replacer l'artiste dans la tradition de la philosophie de l'ordinaire telle que la pense Emerson.

\section{Une poétique de l'accumulation}

La personnalité obsessionnelle de Cornell, dont l'entourage insistait sur la soif inextinguible d'accumuler, en fait un exemple parfait du hoarder, figure américaine du thésauriseur qu'a défini Scott Hering dans son ouvrage The Hoarders: Material Deviance in American Culture. Il est en effet un collectionneur insatiable peut-être avant même d'être un créateur, comme l'illustre son atelier maintes fois photographié, qui montre sa préoccupation constante pour le rangement et l'organisation des objets hétéroclites et sans valeur marchande à partir desquels il créait ses univers oniriques.

Il est difficile de faire une liste exhaustive des objets constituant ses innombrables collections, mais la critique a mis en avant un certain nombre de catégories telles que des images d'oiseaux, de lapins, d'astronomie, témoignant d'un intérêt pour l'exploration du monde depuis sa maison de Utopia Parkway dont il ne s'éloigna jamais. On a souligné que ses collections étaient peut-être une manière pour lui de partager le monde avec son frère malade qui ne pouvait pas quitter la maison familiale. Les collections de Cornell étaient aussi faites d'images de la culture du spectacle : ballets, théâtre, cinéma, souvent illustrées par des photographies de « stars » dont il s'entichait. L'artiste utilisait également des objets ayant perdu leur fonction première : des enveloppes, des cartes, des timbres. Il passait beaucoup de temps à arpenter les rues de New York à la recherche d'objets sans valeur, de collections d'objets achetés dans les dime stores pour quelques centimes, magasins emblématiques de la modernité urbaine du début du $\mathrm{XX}^{\text {ème }}$ siècle : des verres, des bijoux en plastique, des bouts de tissu, des ressorts, des coquillages, des miroirs ou des petits objets décoratifs. Son imaginaire est aussi celui des salles de 
jeu populaires que ses boîtes évoquent très distinctement. Son atelier et sa frénésie de collections font écho à l'avènement moderne d'un surplus d'images et d'objets : ses œuvres présentent une esthétique du quotidien et appartiennent à une culture du divertissement qu'il sublime en objets d'art. Il associe à cet imaginaire de la modernité américaine de nombreuses références à la culture savante et européenne classique. La série des Medici Slot Machines illustre cet agencement de deux univers culturels à priori distincts réunis en une même fantaisie onirique. Untitled (Medici Boy) s'organise ainsi autour de la reproduction du portrait d'un enfant de la famille Medicis, portrait dont la valeur est déjà mise à mal par la reproduction en série mais également par les objets qui l'entourent.

À l'ère de ce que le sociologue Thorstein Veblen appelait la consommation ostentatoire, Cornell porte son attention sur des objets minuscules, autant par leur taille que par leur intérêt ou leur valeur, abaissant le regard de l'artiste vers les objets les plus ordinaires. Ses œuvres-collections se lisent comme des talismans de la culture populaire, des objets ordinaires transformés en mondes miniatures. Il se lance dans son entreprise de collections totales et infinies à un moment où les ÉtatsUnis commencent à s'intéresser au déchet. Susan Strasser explique en effet, dans Waste and Want: A Social History of Trash, que c'est l'avènement de la société de consommation qui a remplacé ce qui était jusqu'alors une culture du recyclage pour ouvrir une nouvelle ère, celle des déchets. Cette ère du détritus qui s'ouvre au début du siècle marque l'avènement des techniques artistiques de collage et d'assemblage. L'exposition de 196I « The Art of Assemblage » au Musée d'art moderne de New York fait retour sur cette esthétique de la modernité américaine, en réunissant les assemblages de Cornell et de Rauschenberg ou Warhol. Cette modernité qui installe les objets de la culture de masse dans la création visuelle se fait jour également en poésie : des déchets du Wasteland chez T.S. Eliot, aux poèmes du quotidien de William Carlos Williams, l'esthétique du déchet ou de l'ordinaire s'installe dans le paysage culturel américain du début du siècle. Le collage, chez Cornell, fait aussi écho aux readymades de Duchamp et notamment à sa Fontaine de I9I4 : si les deux artistes installent l'ordinaire au cœur de leurs préoccupations, il faut souligner que Cornell ne pratique pas l'ironie duchampienne mais cherche bien au contraire à sublimer le banal, à rendre aux objets leur aura perdue, comme l'explique Walter Benjamin, à l'ère de la reproductibilité technique. L'ordinaire semble en effet prendre une coloration différente chez l'européen Duchamp que chez ses contemporains américains pour qui il représente une catégorie esthétique essentielle.

L'art de Cornell a figuré dans les galeries d'avant-garde dès le début de sa carrière et a été considéré comme fondateur par les figures de proue de l'art expérimental américain du XX ${ }^{\text {ème }}$ siècle, il apporte ainsi une illustration très utile aux notions d'avant-garde et de kitsch que le critique Clement Greenberg définit pour fustiger ce qu'il considère comme les dévoiements de l'art à l'ère de la culture de masse. Dans son article de 1939 intitulé « Avant-Garde and Kitsch », Greenberg oppose ces deux 
notions, appelant à un art qui résisterait aux assauts du kitsch, qui ne serait pas facilement consommable. Cornell fonde son travail précisément sur la sublimation du kitsch et des objets sans valeur pour leur assigner une toute autre fonction. Greenberg analyse en effet les boîtes de Cornell comme des cadres vidant leurs objets de leur sens premier (Greenberg I3I).

La multiplication de ces collections suggère l'importance du geste du collectionneur beaucoup plus que celle des objets collectionnés en eux-mêmes ; Cornell souhaitait que son atelier devienne après sa mort un musée ou un atelier expérimental, ce qui montre l'importance et la valeur qu'il conférait à ces objets amassés, indépendamment de leur destin en tant qu'œuvres. L'artiste met en avant une esthétique de l'ordinaire qui célèbre le déchet, travaillant à la manière du chiffonnier que Baudelaire définissait comme la figure même de la modernité : «Tout ce que [la capitale] a dédaigné, tout ce qu'elle a brisé, il le catalogue, il collectionne [...]. Il ramasse, comme un avare, un trésor, ordures qui, remâchées par la divinité de l'industrie, deviendront des objets d'utilité ou de jouissance » (Baudelaire 327). L'art de Cornell repose en effet sur la collecte d'objets trouvés dont la valeur tient à leur accumulation. C'est en effet l'accumulation qui faire renaître ces objets, les faisant passer d'objets ordinaires sans valeur à des trésors offrant une expérience esthétique unique. L'artiste ne cherche pas des images ou des objets spécifiques mais se laisse plutôt guider par le hasard de ses déambulations. L'agencement de ses objets ne suit aucun ordre clair, les objets créant des réseaux issus d'un système de classement proche de l'anecdote sur laquelle Foucault ouvre Les Mots et les choses :

Ce livre a son lieu de naissance dans un texte de Borges. Dans le rire qui secoue à sa lecture toutes les familiarités de la pensée - de la nôtre : de celle qui a notre âge et notre géographie -, ébranlant toutes les surfaces ordonnées et tous les plans qui agissent pour nous le foisonnement des êtres, faisant vaciller et inquiétant pour longtemps notre pratique millénaire du Même et de l'Autre. Ce texte cite « une certaine encyclopédie chinoise » où il est écrit que « les animaux se divisent en : a) appartenant à l'Empereur, b) embaumés, c) apprivoisés, d) cochons de lait, e) sirènes, f) fabuleux, g) chiens en liberté, h) inclus dans la présente classification, i) qui s'agitent comme des fous, j) innombrables, k) dessinés avec un pinceau très fin en poils de chameau, l) et caetera, $m$ ) qui viennent de casser la cruche, $n$ ) qui de loin semblent des mouches ». Dans l'émerveillement de cette taxinomie, ce qu'on rejoint d'un bond, ce qui, à la faveur de l'apologue, nous est indiqué comme le charme exotique d'une autre pensée, c'est la limite de la nôtre : l'impossibilité nue de penser cela. (Foucault 7)

Le moment d'étonnement que décrit Foucault devant ce principe de classification qui paraît absurde montre la fluidité de la notion même de classification : ici la classification met en question ce qui a de la valeur, ce qui est utile ou ce qui ne l'est pas, un peu comme le Musée de Cornell. Il libère les objets de leur signification traditionnelle pour leur réassigner un sens, ou plutôt une infinité de 
sens possibles qu'il appartient au spectateur de définir. La reconfiguration du monde à partir d'objets assemblés dans une trame narrative qui n'est pas explicitée crée une impression de hiéroglyphes : une langue qui semble faire sens mais qui reste pourtant hermétique, la grammaire de cette langue ou sa classification propre étant celle de la série. En effet, les boîtes de Cornell appartiennent très souvent à des séries thématiques : les soap boxes ou les séries sur les oiseaux par exemple.

Les titres de ces boîtes attribuent un sens à ces collages d'objets et d'images, laissant deviner une signification peut-être métaphysique, qui transcende la banalité de l'ordinaire, pour cependant vaciller vers une autre interprétation possible, nous faisant découvrir la « métaphysique de l'éphémère » que recherchait Cornell.

\section{Une métaphysique de l'éphémère}

Cornell intitule l'une de ses œuvres Metaphysic Ephemera: Novalis, plaçant ainsi son esthétique dans la lignée du romantisme allemand, ici mis en relation avec la modernité américaine des objets utilisés et faisant de cette boîte le lieu d'une rencontre entre l'ordinaire ou le banal, et le poétique ou la métaphysique. Il est en cela très proche du Surréalisme, qu'il découvre à New York grâce au galeriste Julien Lévy.

Si Cornell doit, bien sûr, être situé dans le paysage large de la modernité, le contexte spécifique du Surréalisme informe et précipite les débuts de son travail d'artiste. Il découvre ce mouvement et les œuvres de Max Ernst à la galerie Julien Lévy à New York, où il sera rapidement invité à présenter son propre travail, notamment lors de l'exposition Surréalisme en i932. Julien Lévy organisera même une exposition monographique un peu plus tard, intitulée Objects by Joseph Cornell : Minutiae, Glass Bells, Shadow Boxes, Coups d'œil, Jouets Surréalistes, un titre en forme de liste dont la longueur illustre l'esthétique de l'accumulation. New York constitue pour l'artiste la trame de fonds de ses auvres : c'est en flânant le long de ses rues, en s'émerveillant sans cesse devant les images de la modernité de cette ville-monde qu'il construit son imaginaire.

Cornell explique la révélation que constitue pour lui le Surréalisme, mouvement qu'il définit comme un "âge d'or, l'âge de la magie blanche sans laquelle je ne sais pas où je serais aujourd'hui ${ }^{4}$ » (Blair I7). Le roman-collage de Marx Ernst La Femme 100 têtes le marque durablement mais il finira par rejeter cette première association au Surréalisme en insistant sur le fait qu'il se considérait

Ma traduction. 
non pas comme un Surréaliste mais comme « un constructiviste américain » (32). La formule met l'accent sur deux points centraux de son esthétique : l'américanité et la notion de bricolage. Le terme « constructiviste » doit en effet sans doute être entendu comme la mise en avant de son intérêt pour l'agencement formel des multiples éléments de ses boîtes. La formule insiste sur la manière dont ces dernières construisent des mondes en miniature appelant toujours à la redécouverte de nouveaux continents imaginaires, de nouvelles cosmogonies, comme un écho à l'appel transcendantaliste invitant à la redécouverte perpétuelle de la beauté du monde. Cette opposition à l'art européen des Surréalistes illustre le rôle qu'a joué Cornell dans la définition d'une esthétique américaine fondée sur la naïveté du bricoleur. Le collectionneur préserve les objets de sa collection, ce que fait Cornell dans une certaine mesure en préservant ses trouvailles dans des boîtes de bois et de verre, les préservant ainsi de toute détérioration. Mais au-delà, il est essentiellement un bricoleur, qui, à l'inverse du collectionneur, n'hésite pas à détruire, désassembler, casser, pour remonter, ré-agencer et recréer les objets qu'il trouve. Cornell présentait son atelier comme un « laboratoire», un terme qui situe la création artistique du côté de l'expérimentation à partir d'objets et de matériaux de toute nature. Les expérimentations « scientifiques » menées dans ce « laboratoire » sont celles d'une science non-savante, fondée sur le hasard et le bricolage des débris de l'ordinaire. Les objets collectionnés constituaient les outils de Cornell, à la croisée de l'expérimentation et de la création artistique. Cornell est bien un bricoleur tel que le définit Claude Lévi-Strauss : « le bricoleur reste celui qui œuvre de ses mains, en utilisant des moyens détournés, par comparaison avec ceux de 1'homme de l'art » (Strauss 26).

Ces boîtes, que l'artiste définissait comme des jouets, sont constituées d'objets d'une grande banalité qu'elles cherchent à transcender : l'artiste fait d'abord collection de ces objets, puis les détruit pour leur apporter un sens différent. Ce geste destructeur est en réalité un geste de recréation qui redonne vie à l'objet en lui apportant un supplément de sens. Les boîtes se présentent comme un condensé de sens métaphysique, mais cette rencontre entre le réel et la métaphysique est bien sûr éphémère, utopique, et particulièrement bien illustrée dans la série des Soap Bubble Sets où l'éphémère des bulles de savon témoigne de l'impossibilité de capter une vérité métaphysique, et ce en dépit de la forme des boîtes qui est bien un geste de mise sous verre, de manière finale, du sens des choses.

Le bricolage, ou l'agencement aléatoire d'objets épars évoque un monde onirique proche aussi des jeux de l'enfance. Le langage de l'enfance est pour Cornell celui de la tabula rasa, d'une innocence archaïque, d'un paradis perdu sans déchet, où tout objet serait une ouverture sur le rêve et le possible. Lorsque Cornell rejette l'étiquette du Surréalisme pour lui préférer celle de constructiviste américain, il ne semble en réalité que déplacer un même discours, que traduire en termes américains le langage du Surréalisme européen. 


\section{Voir l'ordinaire}

Le rêve et l'utopie dans l'art de Cornell ont été largement soulignés par la critique, mais c'est peut-être la philosophie américaine de l'ordinaire qui permet, en dernier lieu, de situer la pensée du déchet et du recyclage chez cet artiste. Il y a, dans cette œuvre, une insistance sur la notion de découverte du monde qui fait de Cornell, davantage qu'un artiste de la modernité urbaine du début du XX ${ }^{\text {ème }}$ siècle marqué par les expérimentations surréalistes, un artiste profondément américain. Ce qui se joue dans la thématique de l'enfance est la notion américaine de découverte naïve, ce que le critique Tony Tanner appelait « le règne de l'émerveillement » (1977). Cornell réalise la couverture du numéro de la revue surréaliste View en janvier 1943 sur le thème « Americana Fantastica $»^{5}$. Il y superpose des images iconiques de l'Amérique (les chutes du Niagara, des chefs indiens, des trapézistes évoquant l'imagerie populaire des cirques de P.T. Barnum) en suggérant ainsi tout le potentiel fantastique de ce pays, semblant suggérer que le fantastique auquel il cherchait à donner vie se devait d'être ancré dans la culture et la réalité américaine.

En inscrivant le recyclage au cœur de son œuvre, Cornell met en avant la problématique et l'esthétique spécifiquement américaine du banal et de l'ordinaire telle que la définit Emerson. Le philosophe inaugure en effet toute une tradition américaine de l'ordinaire, mais aussi du déchet dans l'essai « The American Scholar », mentionné en début d'article, où il écrit : «j'embrasse le commun, j'explore le familier, le bas, et suis assis à leurs pieds $»^{6}$ (Emerson 67). C'est ce dernier chemin, après celui du contexte moderniste et celui du Surréalisme, le chemin de l'américanité, qui éclaire peutêtre le mieux la pratique du déchet chez cet artiste inclassable. Cornell semble en effet faire sienne la pensée transcendantaliste de l'ordinaire, ce que Emerson suggère dans « Nature » : «Il n'y a pas d'objet si immonde qu'une lumière intense ne rende pas beau $»^{7}$ (Emerson 3I). La lumière intense qui transcende les objets cassés ou sans valeur des boîtes de Cornell provient de la capacité de l'artiste à bricoler, à agencer les objets épars en une cosmologie miniature. L'artiste pratique une philosophie de la synecdoque où le minuscule est une expression de l'infini, un infini de l'émerveillement et de la découverte perpétuelle.

Une reproduction de la revue peut être consultée sur la page suivante : www.bibliopolis.net/cote/viewdata.htm. (page consultée le 20 juin 2020).

6. Traduction Christian Fournier, « Le Savant américain » (564). « I embrace the common, I explore and sit at the feet of the familiar, the low. »

Ma traduction : « There is no object so foul that intense light will not make beautiful. » 
Sandra Laugier explique en effet l'importance de la pensée de l'ordinaire dans la philosophie emersonienne et plus tard dans celle de Stanley Cavell : " Il ne s'agit pas de vanter le sens commun, mais de ramener toute la pensée à l'ordinaire, aux catégories de l'ordinaire : le bas, le proche, qui s'opposent exactement au grand et au lointain, et permettent de 'connaître le sens' de la vie ordinaire » (Laugier 2008, 7). Cornell appartient à cette tradition américaine de la pensée de l'ordinaire : les mondes imaginaires qu'il fabrique sont le fruit de son attention au réel le plus proche et le plus commun. Sandra Laugier rappelle qu'Emerson appelait à une « littérature du pauvre, les sentiments de l'enfant, la philosophie de la rue, la vie domestique, tels sont les sujets du jour » et explique : «Le pauvre, l'enfant, la rue, le domestique : tels sont les nouveaux objets qu'il va falloir, simplement, voir » (Laugier 2002, 9-IO). Ces nouvelles catégories esthétiques sont celles sur lesquelles Cornell construit ses mondes imaginaires, ses boîtes fonctionnant comme des jumelles permettant d'imaginer un ailleurs construit sur les déchets et rebuts de la vie ordinaire.

Le bricoleur Cornell, constructiviste américain auto-proclamé, fait écho à Thoreau, le disciple d'Emerson qui, avec Walden, bricole un ouvrage fait d'anecdotes éparses, un catalogue de notations les plus diverses. Il semble y avoir de nombreux échos entre Cornell et Thoreau : les hiboux de Cornell évoquent ceux de Thoreau, son œuvre Untitled (For Marguerite Blachas) semble faire écho aux notations pratiques de Walden. Cette boîte est creusée dans le Journal d'agriculture pratique si bien que l'on lit, sur la page découpée, le cours des denrées agricoles du 5 au II juillet I9II comme une sorte de réécriture du journal de Thoreau et du compte-rendu minutieux des détails de son quotidien dans la nature. Le geste du bricoleur qu'adopte Cornell s'inscrit dans cette tradition de la construction d'un monde à habiter. Dans Walden, Thoreau présente les détails concrets de sa vie dans les bois mais partage également ses rêves d'un ailleurs. Il écrit ceci :

Lieu et temps à la fois se trouvaient changés, et je demeurais plus près de ces parties de l'univers et de ces ères de l'histoire qui m'avaient le plus attiré. Où je vivais était aussi loin que mainte région observée de nuit par les astronomes. Nous avons coutume d'imaginer des lieux rares et délectables en quelque coin reculé et plus céleste du système, derrière la Chaise de Cassiopée, loin du bruit et de l'agitation. Je découvris que ma maison avait bel et bien son emplacement en telle partie retirée, mais à jamais neuve et non profanée, de l'univers. S'il valait la peine de s'établir en ces régions voisines des Pléiades ou des Hyades, d'Aldébaran ou d'Altaïr, alors c'était bien là que j'étais, ou à une égale distance de la vie que j'avais laissée derrière. (Thoreau 86)

Cette description pourrait tout à fait s'appliquer aux boîtes de Cornell, qui présentent une astronomie rêvée, ce qui permet d'établir une filiation forte entre cet artiste moderniste et les premiers penseurs de la philosophie de l'ordinaire qu'étaient Thoreau et Emerson. Les boîtes de Cornell sont 
des explorations qui fonctionnent comme des catalogues naturalistes et prennent un sens particulièrement américain dans la mesure où les catalogues naturalistes, relevés topographiques et géologiques revêtaient une importance cruciale pour les colons. Les cartographies et mises sous verre des objets de l'Amérique contemporaine font écho à une histoire de la cartographie et du catalogue propre aux États-Unis. Cette histoire appartient aussi à celle de l'art américain telle que commence à la présenter le peintre Charles Wilson Peale dans son fameux tableau The Artist in his Museum (I822). ${ }^{8}$ Le tableau laisse entrevoir les collections naturalistes et artistiques du peintre, les cadres et boîtes d'exposition préfigurant les boîtes de Cornell, à la différence cependant que la taxonomie de Cornell se situe du côté du rêve beaucoup plus que de la science.

Les oiseaux de Cornell évoquent aussi ceux de l'ornithologue et naturaliste du début du $\mathrm{XIX}^{\text {ème }}$ John James Audubon : on note une même concentration sur la couleur et la composition, une mise en avant de la beauté de l'oiseau qui appelle à l'émerveillement. Les boîtes fonctionnent comme des répliques amateures de ces investigations scientifiques des oiseaux du continent. Les boîtes de Cornell semblent bien être des avatars des cabinets naturalistes, ceux par exemple du Muséum d'histoire naturelle de Paris qui précipita la pensée d'Emerson vers une pensée de la nature et du monde fondée sur l'idée d'un ordre transcendantal (Brown). Emerson décrit sa découverte du Muséum en composant des listes qui font du texte un cabinet de curiosités, sorte de préfiguration des collections de Cornell qui fonctionnent aussi sur le mode de la liste, itération à l'infini d'objets épars, dont chaque arrangement constitue un monde en miniature.

La figure du bricoleur est très utile pour penser le travail de Cornell car elle met en avant la notion d'un art non-savant, non-scientifique, fait des déchets du monde ordinaire, cherchant à réenchanter le réel. Elle donne à voir un art sans autorité, où le goût et la technique n'ont pas d'autre ligne directrice que le hasard ou l'inconscient.

Les œuvres de Cornell évoquent les cabinets de curiosité, les musées d'histoire naturelle et notamment celui de Paris tel que décrit par Emerson, des coffres recelant des trésors, des encyclopédies - autant de mondes clos qui tentent de circonscrire le monde. Dans ses notes, Cornell décrit le processus d'association libre sur lequel reposait pour lui la création artistique : « débordement rapide d'expérience [...], encombrement d'incidents et d'expériences, chemins qui s'ouvrent toujours plus, menant toujours plus loin. Possibilités de croisements (d'expériences) incroyablement riches, flux

Le tableau est visible sur le site de la Pennsylvania Academy of the Fine Arts : pafa.org/museum/collection/item/ artist-his-museum (page consultée le 20 juin 2020). 
incessant et entrelacs d'expériences originales $\gg{ }^{9}$ (Blair 5I). Cette description du processus créatif en des termes qui évoquent la découverte d'un espace physique rappelle les catalogues des muséums ou l'organisation des encyclopédies. Il faut cependant souligner que cette taxonomie ne semble pourtant rentrer dans aucun cadre précis, ces références étant toujours en flux, se croisant et se décroisant au profit d'une expérience de ce que la critique Lindsay Blair, appelle en citant ce passage, « l'ordre spirituel » de Cornell (I998).

Cet ordre spirituel est peut-être, essentiellement, celui de l'américanité. Cornell s'approprie une méthode européenne, le Surréalisme, mais la traduit en termes américain, s'inscrivant dans une tradition qui remonte jusqu'au transcendantalisme. Il oppose à l'ironie surréaliste l'innocence américaine du wonder, de l'émerveillement américain défini par Tony Tanner et ce faisant nous invite à découvrir un monde idéal, créé à partir des objets abandonnés de la réalité.

\section{OUVRages CITÉS}

BANASH, David. Collage Culture: Readymades, Meaning, and the Age of Consumption. Amsterdam : Rodopi, 20I3.

BAUDELAIRE, Charles. Paradis artificiels in Euvres complètes. Paris : Gallimard La Pléiade, I96I.

BAUDRILLARD, Jean. Le Système des objets. Paris : Gallimard, I968.

BERDET, Marc. « Chiffonnier contre flâneur. Construction et position de la Passagenarbeit de Walter Benjamin ». Archives de Philosophie 75:3 (juillet-septembre 20I2). 425-447.

BLAIR, Lindsay. Joseph Cornell's Vision of Spiritual Order. Londres : Reaktion Books, 1998.

BROWN, Lee Rust. The Emerson Museum: Practical Romanticism and the Pursuit of the Whole. Cambridge, MA : Harvard University Press, 1997.

CAVELL, Stanley. « The World as Things: Collecting Thoughts on Collecting »". Contemporary Collecting: Objects, Practices, and the Fate of Things. Eds. Kevin M. Moist and David Banash. Lanham, MD : Scarecrow Press, 20I3.

CAWS, Mary Ann. Joseph Cornell's Theater of the Mind: Selected Diaries, Letters, and Files. Londres : Thames and Hudson, 2000.

9. Ma traduction : "rapid overflow of experience [...], overcrowding of incident and experience ever opening paths leading ever farther afield. Unbelievably rich cross-indexing (of experience) the ceaseless flow and interlacing of original experience". 
CRAN, Rona. Collage in Twentieth Century Art, Literature and Culture: Joseph Cornell, William Burroughs, Frank O'Hara and Bob Dylan. Farnham: Ashgate, 2014.

DEBARY, Octave. De la Poubelle au musée. Une anthropologie des restes. Paris : Creaphis, 2019.

EDWARDS, Jason and Stephanie L. TAYLOR (eds.). Joseph Cornell: Opening the Box. Berne : Peter Lang, 2007.

ELSNER John, and Roger CARDINAL (eds.). Cultures of Collecting. Londres : Reaktion Books, I994.

FOUCAULT, Michel. Les Mots et les choses. Paris : Gallimard I966.

FOURNIER, Christian. «Le Savant américain », traduction de « The American Scholar ». Critique : La Nouvelle Angleterre, dirigé par C. Chauviré n 54 I-542, juin-juillet, I992. 548-566.

GREENBERG, Clement. The Collected Essays and Criticism, Volume 1: Perceptions and Judgments 19391944. Ed. John O’BRIAN. Chicago et Londres : University of Chicago Press, 1986.

HERING, Scott. The Hoarders: Material Deviance in American Culture. Chicago : University of Chicago Press, 20I4.

LAUGIER, Sandra. «L'Ordinaire transatlantique : de Concord à Chicago en passant par Oxford ». L'Homme I87-I88 (2008) : 649-768. URL : https://doi.org/I0.400o/lhomme.29239 (page consultée le 20 juin 2020).

LAUGIER, Sandra. «Emerson : Penser l'ordinaire ». Revue française d'études américaines 9I (février 2002) : $43-60$.

LEVI-STRAUSS, Claude. La Pensée sauvage. Paris : Plon, 1962.

PICON, Raphaël. Emerson. Le Sublime ordinaire. Paris : CNRS Editions, 2015

PORTE, Joel and Saundra MORRIS (dir.). Emerson's Prose and Poetry. New York et Londres : Norton, $200 \mathrm{.}$.

RONDA, Bruce. The Fate of Transcendentalism: Secularity, Materiality, and Human Flourishing. Athens, GA : University of Georgia Press, 2017.

SOLOMON, Deborah. Utopia Parkway: The Life and Work of Joseph Cornell. New York : Farrar, Straus and Giroux, 1997.

STRASSER, Susan. Waste and Want: A Social History of Trash. New York: Henry Holt, 2000.

TANNER, Tony. The Reign of Wonder: Naivety and Reality in American Literature. Cambridge : Cambridge University Press, 1977.

THOREAU, Henry David. Walden ou la vie dans les bois. Trad. L. Fabulet. Paris : NRF, I922.

VEBLEN, Thorsten. The Theory of the Leisure Class. Londres : Penguin Classics, 1994.

WALDMAN, Diane. Joseph Cornell Master of Dreams. Ann Arbor, MI : The University of Michigan, Press, 2002. 